\section{Atopische Töchter sind trauriger}

\begin{abstract}
Mehrere Studien haben bereits den Zusammenhang zwischen atopischen und psychiatrischen Erkrankungen untersucht. Eine große, retrospektive Untersuchung aus Finnland liefert neue Hinweise auf eine Verbindung zwischen Allergien und Depressionen.
\end{abstract}

K liniker der Universität Oulu erhoben mit Fragebögen die Daten von 4.068 im Jahre 1966 geborenen Bewohnern der nordfinnischen Provinzen Oulu und Lappland. Abgefragt wurden die ärztlich gesicherte Diagnose einer Depression sowie Angaben zur Eigenund Familienanamnese hinsichtlich atopischem Ekzem, allergischer Rhinitis und Asthma. Zusätzlich wurde ein Pricktest mit den vier in Finnland wichtigsten Allergenen durchgeführt.

Von den befragten 2.125 Frauen waren $4,9 \%$, von den befragten 1.943 Männern waren 2,8\% schon einmal als depressiv diagnostiziert worden. 6,8\% der Frauen mit atopischen Eltern waren depressiv im Gegensatz zu 3,5\% der Teilnehmerinnen aus diesbezüglich gesunden Familien $(\mathrm{p}<0,001)$. Daraus ergab sich ein um den Faktor 2,1 erhöhtes
Risiko für Depressivität, falls beide Eltern Atopiker waren. Litten außer den Eltern auch die Frauen selbst unter Allergien, so erhöhte sich das Depressionsrisiko um den Faktor 4,5. Dabei hatten die Mütter größeren Einfluss als die Väter (Odds Ratio: 4,1 bei mütterlicher und eigener Allergie, 2,7 bei väterlicher und eigener Allergie). Bei den Männern zeigte sich keine Korrelation zwischen Depressivität und Allergien.

Die Untersucher interpretieren das gemeinsame Auftreten von Depression und atopischer Familienanamnese im Sinne von mütterlicherseits vererbten genetischen Faktoren. Es bleibt kritisch anzumerken, dass die Daten zur „Depressivität “ auf einer einzigen retrospektiven Frage basieren, wobei die Autoren auch nicht zwischen endogener und exogener Depression differenzieren.

\title{
Asthmarisiko: Sektio caesarea ungünstig
}

\section{Der Kaiserschnitt hat einen geringen, aber nachweisbaren Effekt auf die Asthmahäufigkeit. Das ergab eine dänische Studie im vorigen Jahr, referiert in Allergo J 2003; 12: 162. Eine große schwedische Analy- se untermauert jetzt dieses Ergebnis und noch mehr ...}

D ie Häufigkeit von Schnittentbindungen hat in manchen europäischen Ländern inzwischen die 20\%Marke erreicht. Kaiserschnitt-Babys zeigen gegenüber Kindern, die auf natürlichem Wege zur Welt kamen, eine verzögerte mikrobielle Darmkolonisation. Auch ist die Darmflora allergischer Kinder anders zusammengesetzt als bei Gesunden. So verwundert es nicht, dass über die Sectio caesarea als mögliche Ursache von Allergien spekuliert wird.

Schwedische Kinderärzte der Universitäten Lund und Umeå suchten in Geburtenregistern und Krankenhaus- entlassungsstatistiken der Jahre 19841996 nach Sektiogeburten und Klinikaufenthalten wegen Asthma oder Gastroenteritis von Kindern älter als ein Jahr. Dabei berücksichtigten sie nur normalgewichtige, reife Neugeborene mit gesunden Müttern ohne Mehrlingsgeburten.

Von 13.058 Asthmapatienten waren $8,6 \%$, von 20.377 Kindern mit Durchfallerkrankungen waren $8,5 \%$ per Kaiserschnitt zur Welt gekommen. Dagegen waren unter den 637.901 Kindern, die im Untersuchungszeitraum nie stationär aufgenommen worden wa-

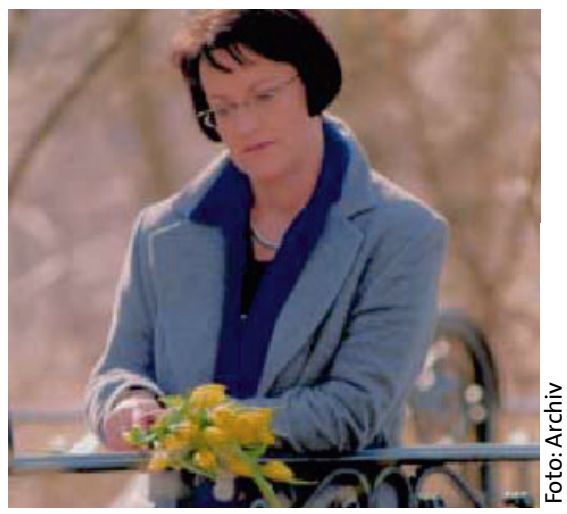

Leidet sie unter Depressionen, weil sie Allergikerin ist?

Fazit: Finninnen mit positiver Familien- oder Eigenanamnese bezüglich atopischer Erkrankungen haben ein erhöhtes Risiko, depressiv zu werden. $\mathrm{Ob}$ diese Daten aufwendige genetische Untersuchungen rechtfertigen oder ob hier eher Psychologen gefragt sind, sei dahingestellt.

Timonen $\mathbf{M}$ et al. Presence of atopy in first-degree relatives as a predictor of female proband's depression: results from the Northern Finland 1966 Birth Cohort. J Allergy Clin Immunol 2003; 111: 1249-54

ren, nur 6,9\% Kaiserschnitt-Babys. Daraus ergibt sich ein 1,31-fach erhöhtes Risiko $(p<0,001)$ für Sektio-Kinder, an Asthma oder Gastroenteritis zu erkranken. Der Befund, dass normal geborene Geschwister von KaiserschnittKindern kein erhöhtes Risiko für Asthma und nur ein 1,12-fach erhöhtes Risiko $(\mathrm{p}<0,05)$ für Durchfallerkrankungen hatten, spricht für einen direkten Zusammenhang zwischen der Schnittentbindung und Asthma bzw. Gastroenteritis.

Fazit: Kinder, die per Kaiserschnitt zur Welt kommen, haben ein um 30\% erhöhtes Risiko, eine stationäre Behandlung wegen Asthma oder Gastroenteritis zu benötigen.

cl

Håkansson S et al. Caesarean section increases the risk of hospital care in childhood for asthma and gastroenteritis. Clin Exp Allergy 2003; 33: 757-64 